\title{
Graphical analysis of bra size calculation procedures
}

Bra size calculation procedures

\author{
M.C.M. Wright \\ Institute of Sound and Vibration Research, \\ University of Southampton, UK
}

Received October 2000

Keywords Garments, Measurement

Accepted August 2001

Abstract A widely used procedure for calculating bra size from body measurements is analysed graphically. It is shown that arbitrarily small variations in the body measurements can cause a difference of up to three cup sizes in the calculated bra size. Some implications are discussed and improved procedures suggested.

\section{The calculation procedure}

The rise of internet shopping has brought an increasing need for consumers to measure themselves rather than consulting experienced fitters. An internet search has revealed that variations on the following procedure for calculating bra sizes is quoted on many retail sites and elsewhere:

(1) Measure around the ribcage just under the bust in inches.

(2) If the result is even add four, otherwise add five, this is the band size.

(3) Measure around the bust at its fullest point.

(4) Subtract the result of (2) from the result of (3).

(5) Convert this number to a letter, this is the cup size.

There is an implicit assumption that the results of steps (1) and (3) must be integers. It will be assumed in what follows that this is the result of a rounding to the nearest integer, so that the range from 0.5 to 1.499 ...rounds to 1 , etc. Variations are found in the letter system used in step (5), but these are immaterial to the discussion that follows. For definiteness the system shown in Table I, which is common in the UK, will be used.

Mathematically the procedure defines a mapping from two continuous variables $x$ and $y$, the actual ribcage and bust sizes, to two discrete variables $X$ and $Y$, the resulting band and cup size. This mapping can be written

$$
\begin{gathered}
X=2\lfloor(\lfloor x+1 / 2\rfloor+5) / 2\rfloor, \\
Y=\lfloor y+1 / 2\rfloor-2\lfloor(\lfloor x+1 / 2\rfloor+5) / 2\rfloor,
\end{gathered}
$$

where $\lfloor x\rfloor$ is the greatest integer less than or equal to $x$. Hence or otherwise, the domain in $(x, y)$ space occupied by each $(X, Y)$ pair can be found. This is plotted in Figure 1 with $Y$ expressed as a letter.

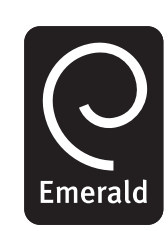

International Journal of Clothing Science and Technology, Vol. 14 No. 1, 2002, pp. 41-45. (C) MCB UP Limited, 0955-6222 DOI 10.1108/09556220210420327 
IJCST

14,1

42

As can be seen from Figure 1, each size (i.e. each distinct $(X, Y)$ pair) occupies a rectangular domain in $(x, y)$ space, 2 inches wide and 1 inch tall. The domains corresponding to a particular cup size, however, are not adjacent, whereas there exist pairs of domains whose cup sizes differ by two which are horizontally adjacent. By the same token there exist pairs of domains whose cup sizes differ by three which are diagonally touching. This means that arbitrarily small errors in $x$ and $y$ could, if they caused the measurement to cross the relevant diagonal boundary between domains, change a predicted size from an A-cup to a D-cup, for example.

The cup size is suppposed to describe the difference between the bust and ribcage sizes. The points on a line inclined at $45^{\circ}$ in $(x, y)$ space have a constant difference between $x$ and $y$. It is impossible, however, to draw such a line on Figure 1 so that it passes through the domains corresponding to any one cup size for more than half of its length, if the line is continued far enough. Coincidentally it is often claimed that $50 \%$ of women in the UK wear the wrong size of bra, although a literature search has failed to reveal any published research to support this statistic. Note also that although cup sizes go up in one inch steps the difference between the smallest and largest values of $x-y$ to be assigned to the same cup size is three inches.

\section{An alternative procedure}

The cause of these anomalies can be seen in Equation (2). The ribcage size $x$ is rounded twice, once (implicitly) when measured and then again when made even. The cup size $Y$, therefore, is the difference between two rounded quantities, which allows the rounding errors to accumulate. That error is plus or minus half an inch for the first term, and plus or minus one inch for the second, leading to an accumulated error of plus or minus one and a half inches, corresponding to the three inch range in $x-y$ values.

The situation can be improved by modifying the procedure as follows:

(1) Measure around the ribcage just under the bust in inches.

(2) Add five to the result.

(3) Measure around the bust at its fullest point rounding up to the next integer.

(4) Subtract the result of (2) from the result of (3).

(5) Convert this number to a letter, this is the cup size.

(6) If the result of (2) is odd subtract one, this is the band size.

Table I.

Key to cup size letters

\begin{tabular}{lllllll}
\hline-1 & 0 & 1 & 2 & 3 & 4 & 5 \\
$\mathrm{AA}$ & $\mathrm{A}$ & $\mathrm{B}$ & $\mathrm{C}$ & $\mathrm{D}$ & $\mathrm{DD}$ & $\mathrm{E}$ \\
\hline
\end{tabular}




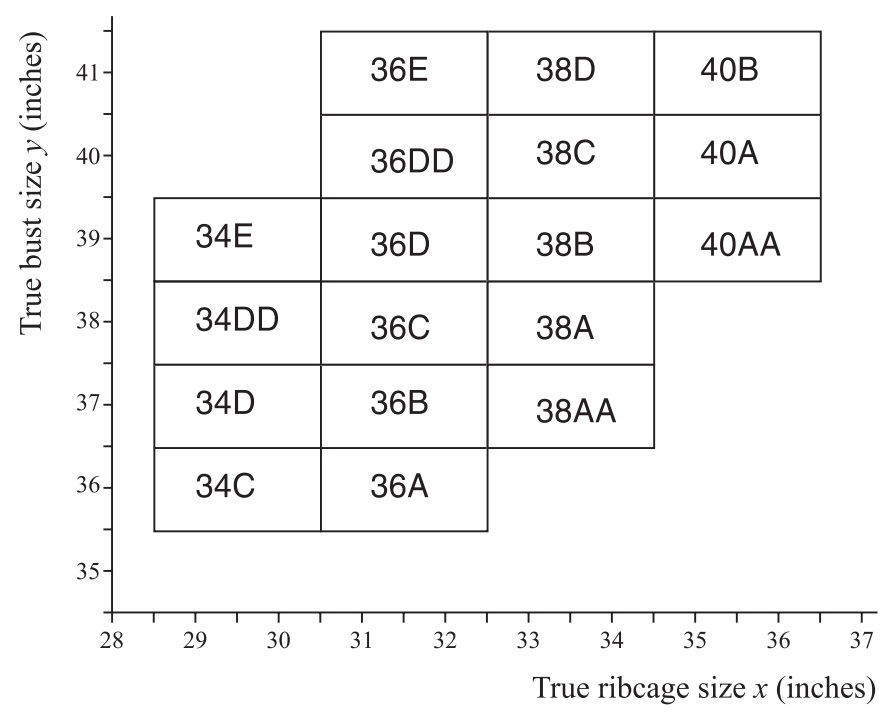

This corresponds to the following mapping:

$$
\begin{gathered}
X=2\lfloor(\lfloor x+1 / 2\rfloor+5) / 2\rfloor, \\
Y=\lfloor y+1\rfloor-\lfloor x+1 / 2\rfloor-5,
\end{gathered}
$$

which is plotted in Figure 2.

Now all the domains corresponding to a particular cup size are at least diagonally touching and it is possible to draw a continuous $45^{\circ}$ line which passes only through the domains corresponding to a single cup size. The cup size is still the difference of two rounded quantities, but the error in the second is reduced because now it is only rounded once. The range of $y-x$ values to be assigned to the same cup size therefore becomes two inches, as can be verified from Figure 2.

For completeness it may be worth pointing out that it is possible to completely remove all error accumulation by arranging for the difference operation to occur before any rounding. This could be achieved if, for example, a disposable paper tape were used to make the measurements, on which marks were made to indicate its position when placed around the ribcage and bust. The calculation procedure would then be:

(1) Measure the distance from the beginning of the tape to the first mark.

(2) If it is even add four, if it is odd add five, this is the band size.

(3) Measure the difference between the marks rounding up to the next integer.

(4) Subtract five and convert to a letter.
Bra size calculation procedures

Figure 1. Domain map for first procedure 
IJCST

14,1

\section{4}

Figure 2.

Domain map for second procedure

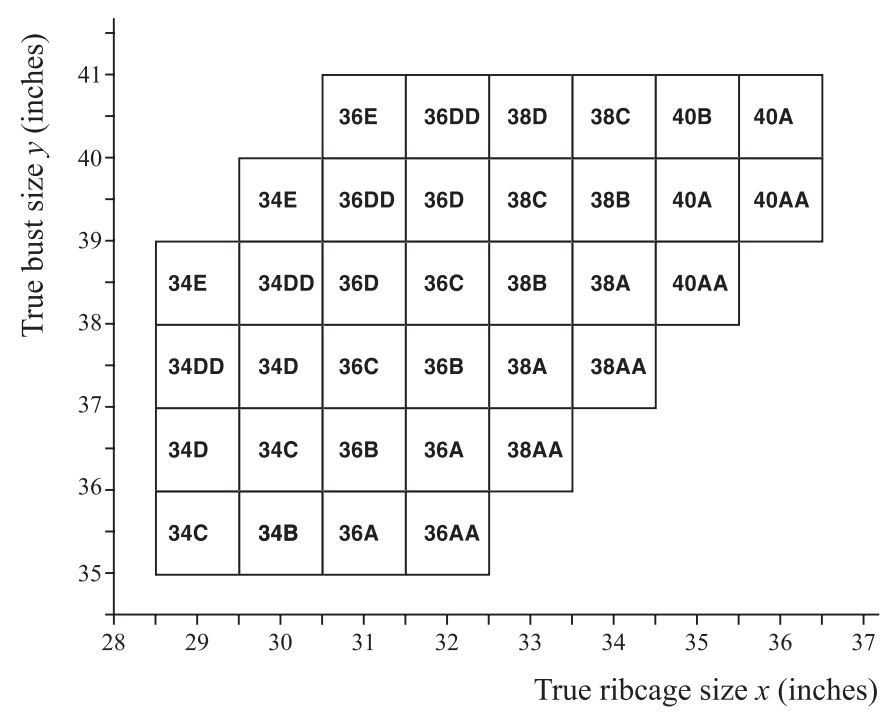

The corresponding mapping is

$$
\begin{gathered}
X=2\lfloor(\lfloor x+1 / 2\rfloor+5) / 2\rfloor, \\
Y=\lfloor y-x+1\rfloor-5,
\end{gathered}
$$

which is plotted in Figure 3.

This reduces the range of $y-x$ over a cup size to one inch, albeit at the expense of a more involved procedure.

\section{Discussion}

In practice consumers will usually find their preferred size by trial and error, although this may not always be the case if the 50 per cent statistic mentioned above does have any basis in fact. The success of any procedure can only be judged by the likelihood of its predicting the same size as would be chosen by trial and error, and no such procedure can be as successful as an experienced fitter. But with the rise of internet shopping the reliance on such procedures seems likely to grow. The graphical analysis carried out above suggests that the second procedure should have a greater likelihood of success than the first.

Naturally the manufacturers' implementation of sizes will be a factor in the success of any procedure, but generally this will only affect the location and scaling of the patterns in Figures 1 and 2, not the relative positions of the domains therein. In particular it is hard to conceive of a rational manufacturing strategy for which the first procedure is more suitable than the second. 


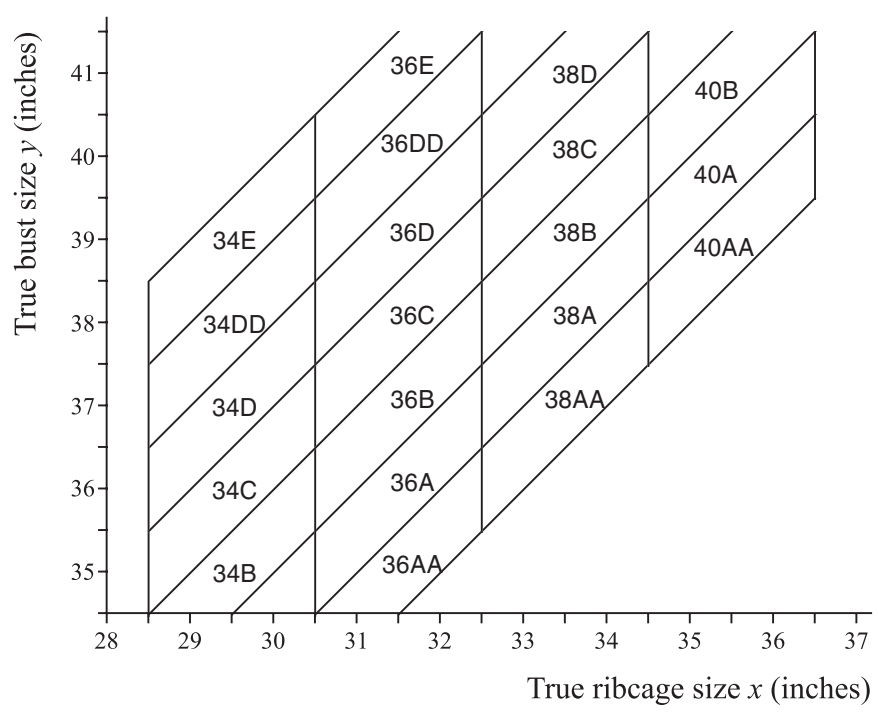

Bra size
calculation
procedures

45

Figure 3.

Domain map for third procedure

The implications of this analysis go beyond manufacturer/retailer/consumer relationships. Before reconstructive or cosmetic breast surgery the postoperative bra size is predicted, and the mappings discussed above can be signigicant (Kanhai, 1999). Furthermore, medical statistics relating, for instance, incidence of cancer to breast size may be based on census data which asks for cup size. The procedure used to calculate that cup size could then have implications for the interpretation of such statistics.

\section{References}

Kanhai, R.C.J. and Hage, J.J. (1999), "Bra cup size depends on band size", Plastic and Reconstructive Surgery, Vol. 104 No. 1, pp. 300. 